\title{
Asimetría mandibular en radiografías de niños no sindrómicos con y sin hendidura labio palatina en Bucaramanga, Colombia.
}

Mandibular asymmetry in radiographies from non-syndromic children with and without cleft lip and palate in Bucaramanga, Colombia.

Javira La Rosa ${ }^{1, a}$, Mónica Restrepo ${ }^{1, a}$, Ethman Ariel Torres 1,b, Martha Juliana Rodríguez ${ }^{1, c}$. RESUMEN

Objetivo: determinar si existen diferencias en el crecimiento y desarrollo mandibular que evidencien una mayor prevalencia de asimetría en radiografías de pacientes con hendidura labio palatina. Material y métodos: se realizó un estudio observacional analítico de corte transversal con una muestra de radiografías panorámicas y cefálicas laterales de 130 pacientes de Bucaramanga que asistieron al servicio de ortodoncia de la Universidad Santo Tomás. Se evaluaron $64(49,2 \%)$ hombres y $66(50,8 \%)$ mujeres en edades comprendidas entre 6 a 16 años con una edad promedio de 10,6 $\pm 3,1$ años. Se definieron puntos anatómicos en las radiografías para realizar las mediciones en los dos grupos. Se aplicó la prueba t de Student y el análisis multivariado de ANOVA con una significancia de $p \leq 0,05$. Resultados: se encontró una diferencia estadísticamente significativa en la simetría de los pacientes con labio y paladar hendido $(\mathrm{p}=0,0340)$, así como una menor altura de la rama izquierda representada por el plano cefalométrico Co-Inc $(\mathrm{p}=0,0414)$ y del cuerpo mandibular por Co-Gn $(\mathrm{p}=0,0094)$ y Go - Pog ( $\mathrm{p}=0,0015)$. Conclusiones: el tamaño del cuerpo mandibular representado por las medidas tomadas en radiografías panorámicas y cefálicas laterales (Go-Pog, Co-Gn) de pacientes con labio y paladar hendido es más pequeño que el de los pacientes sin hendidura.

Palabras clave: fisura del paladar, labio leporino, mandíbula, cefalometría. 


\section{ABSTRACT}

Objective: assess any difference in growth and development of mandible in radiographs from cleft lip and/or palate patients. Methods: an analytical cross-sectional study was conducted with a sample of 130 panoramic and cephalic radiographies from patients who attended the orthodontic service at Universidad Santo Tomás. Sixty-four $(49.2 \%)$ men and $66(50.8 \%)$ women with an average age of $10.6 \pm 3.1$ years were assessed. Anatomical points were defined on the radiographies and the measurements were performed in the two groups. The Student t-test and the multivariate ANOVA analysis were applied. A $\mathrm{p} \leq 0.05$ was considered as statically significant. Results: a statistically significant difference was found in the symmetry of patients with cleft lip and palate $(\mathrm{p}=0.0340)$, as well as a lower height of the left branch represented by the cephalometric plane Co-Inc $(\mathrm{p}=0.0414)$ and the mandibular body by Co - Gn ( $\mathrm{p}=0.0094)$ and Go - Pog $(\mathrm{p}=0.0015)$. Conclusions: the size of the mandibular body represented by the measurements taken in panoramic and lateral cephalic radiographs (Go-Pog, Co-Gn) of patients with cleft lip and palate is smaller than that of patients without clefts.

Keywords: cleft palate, cleft lip, mandible, cephalometry.

\section{INTRODUCCIÓN}

La hendidura labio palatina se origina por deficiencia en la fusión de los procesos nasales y maxilares a partir de la sexta semana embrionaria(1). Es la malformación craneofacial más frecuente y el segundo defecto de nacimiento después del Síndrome de Down, con una prevalencia de 1 por 1000 nacidos vivos, con amplia variabilidad en lo geográfico y social $(2,3)$. Su etiología es considerada poligenética y multifactorial (4). Los avances en genética y biología molecular han permitido entender mejor la embriología y el crecimiento craneofacial y han mostrado como las variaciones en genes homeobox, al alterar el mecanismo de migración de las células de la cresta neural y las relaciones epitelio-mesénquima, pueden presentar hendiduras labiales o palatinas pero también afectar todo el crecimiento craneofacial puesto que todas las estructuras provenientes de los arcos faríngeos, son regulados por los mismos genes $(5,6)$. Es importante conocer si los pacientes con labio y/o paladar hendido no sindrómico pueden presentar otras alteraciones craneofaciales posiblemente relacionadas por el efecto modulador de estos mismos genes. Una de estas características craneofaciales es el tamaño y la forma del maxilar inferior que se relaciona con la presencia de la simetría facial.

Los pacientes con labio y paladar hendido pueden presentar diferentes patrones craneofaciales y esto varía según el tipo de hendidura, los efectos de las cirugías, el tratamiento recibido y el crecimiento individual de cada paciente $(3,7)$. Sin embargo, en muchos de ellos se encuentra una maloclusión clase III esquelética con retrusión del maxilar respecto a la base de cráneo, micrognatismo maxilar, rotación maxilar hacia abajo y atrás y dientes superiores en palatoversión (8). A nivel mandibular, se observa un perfil cóncavo, dientes inferiores en linguoversión, $\mathrm{y}$ un menor tamaño, cuando se compara con pacientes sin hendidura, además de una mayor prevalencia de asimetría facial (8-10). Este fenotipo mandibular, que según Miller y colaboradores difiere significativamente de los controles, se relaciona con el genotipo encontrado en estos pacientes, la posición anteroposterior de la mandíbula en los pacientes con labio y paladar hendido no sindrómico se ha relacionado con SNA11, IRF6, MSX1 y MAFB (11). Estas características del crecimiento mandibular se han encontrado en las razas caucásicas, asiáticas y en una muestra de Tanzania $(8,9,11,12)$.

Al existir diferencias en la prevalencia y el tipo de hendiduras según la razas $(3,4)$; este trabajo pretendió conocer cuál es la morfología y asimetría mandibular de los pacientes con labio y paladar hendido (LPH) en una muestra de la población colombiana dado que los estudios en genética de diferentes poblaciones y características craneofaciales indican que estos patrones cambian de una población a otra y al no contar con suficientes datos de la población colombiana en este aspecto, es importante profundizar sobre esta temática. El objetivo de este estudio fue determinar si existen diferencias en el crecimiento y desarrollo mandibular que evidencien una mayor prevalencia de la asimetría y menor tamaño mandibular en niños 
con hendidura labio palatina comparados con sus controles.

\section{MATERIAL Y MÉTODOS}

Se realizó un estudio observacional de corte transversal con el fin de determinar posibles diferencias en la morfología mandibular de los niños con labio y/o paladar hendido en comparación con niños sanos. Se tomó una muestra de radiografías panorámicas y cefálicas laterales provenientes del Centro de Imágenes Diagnósticas de la Universidad Santo Tomás (Colombia); el equipo utilizado para la toma de radiografías fue FONA Xpan 3Dplus. El tamaño de muestra se calculó mediante el paquete estadístico Epi-Info 6,04 al considerar un poder del $80 \%$, un nivel de confianza del $95 \%$ y una prevalencia de asimetrías del $50 \%$ en personas con LPH, por lo cual 65 individuos no expuestos y 65 expuestos permitían detectar asociaciones estadísticamente significativas. El tipo de muestreo fue por conveniencia y la muestra estuvo conformada por radiografías de 130 pacientes en edades entre 6 a 16 años, 64 (49,2\%) de los cuales fueron hombres. La población la constituyeron dos grupos:

Grupo 1: radiografías panorámicas y cefálicas laterales de 65 pacientes que presentaban hendidura labio palatina no sindrómica. De estos 65 pacientes, $38(58,5 \%)$ presentaban hendidura unilateral y 27 $(41,5 \%)$ hendidura bilateral.

Grupo 2: radiografías panorámicas y cefálicas laterales de 65 pacientes que no presentaban hendidura labio palatina y sin antecedentes familiares de hendidura.

Se excluyeron del estudio las radiografías de pacientes que hubieran sufrido traumatismos faciales, radiografías en las que no se ubicaran adecuadamente y con nitidez los puntos usados para las mediciones debido a poco contraste o imágenes dobles, y radiografías de pacientes que presentaran pérdida de más cinco estructuras dentarias. Las variables consideradas en el estudio se recopilaron en un instrumento diseñado para este fin que incluía:

- Variables sociodemográficas: sexo y edad.

- Variables cualitativas: presencia o no de hendidura labio palatina, tipo de hendidura palatina, localización de la hendidura y presencia de asimetría facial.

- Variables cefalométricas:

En la radiografía panorámica se trazaron los puntos anatómicos Co, Inc y Go, y se midieron los planos según metodología propuesta por Kjellberg y colaboradores; estos se mencionan a continuación: (13)

- Plano Co - Inc: medida lineal sobre radiografía panorámica desde el punto Condilion $(\mathrm{Co})$ hasta Incisura (Inc) que representa la altura del cóndilo.

- Plano Inc - Go: medida lineal sobre radiografía panorámica desde el punto Incisura (Inc) hasta Gonión (Go) que representa la altura de la rama.

- Plano Co - Go: medida lineal sobre radiografía panorámica desde el punto Condilion $(\mathrm{Co})$ hasta Gonión (Go) que representa la altura total de la rama mandibular (Figura 1).

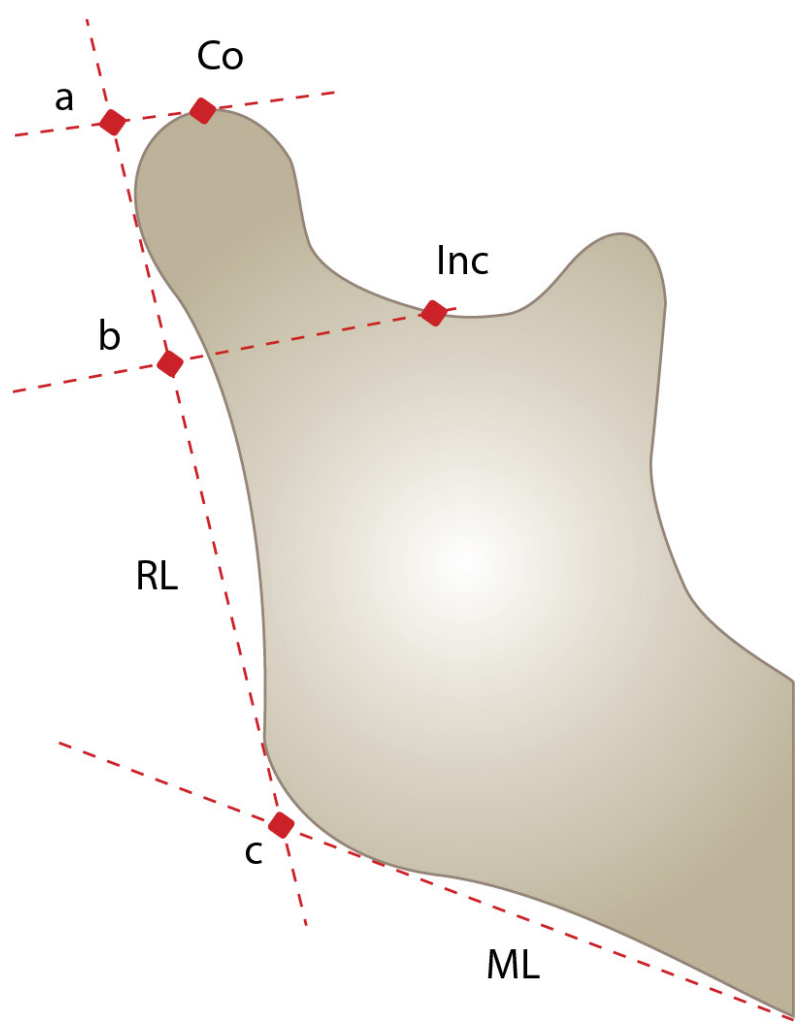

Figura 1. Análisis de la radiografía panorámica según Kjellberg $\mathrm{H}$ y colaboradores ${ }^{13}$. Inc $=$ Incisura, $\mathrm{Co}=$ Condilion, $\mathrm{b}=$ Altura del cóndilo, $\mathrm{RL}=$ Altura de la rama $\sin$ incluir la altura del cóndilo. 
En la radiografía cefálica lateral se trazaron los puntos anatómicos Ar, Go, Po, Gn y se midieron los siguientes planos, según la metodología reportada por Kyrkanides y Richter (14).

- Plano Ar - Go: medida lineal sobre radiografía cefálica lateral desde el punto Articular (Ar) hasta Gonión (Go) que representa la altura de la rama.

- Plano Go - Pog: medida lineal sobre radiografía cefálica lateral desde el punto Gonión (Go) hasta Pogonión (Pog) que representa la longitud del cuerpo mandibular.

- Plano Co - Gn: medida lineal sobre radiografía cefálica lateral desde el punto Condilion (Co) hasta Gnation (Gn) que representa la diagonal del cuerpo de la mandíbula (Figura 2).

Los trazos y las mediciones fueron realizadas de forma manual por dos de los investigadores (JLR, MR) previamente capacitados con un Especialista en Ortodoncia. La calibración inter e intraexaminador se realizó bajo las mismas condiciones en que se evaluarían las radiografías. Se estimó el Coeficiente de Correlación Intraclase (CCI) y se tuvo en cuenta que un valor superior a 0,60 indicaba una adecuada calibración. Los CCI encontrados en la evaluación interexaminador fueron 0.8467 y 0.7006 , en tanto que

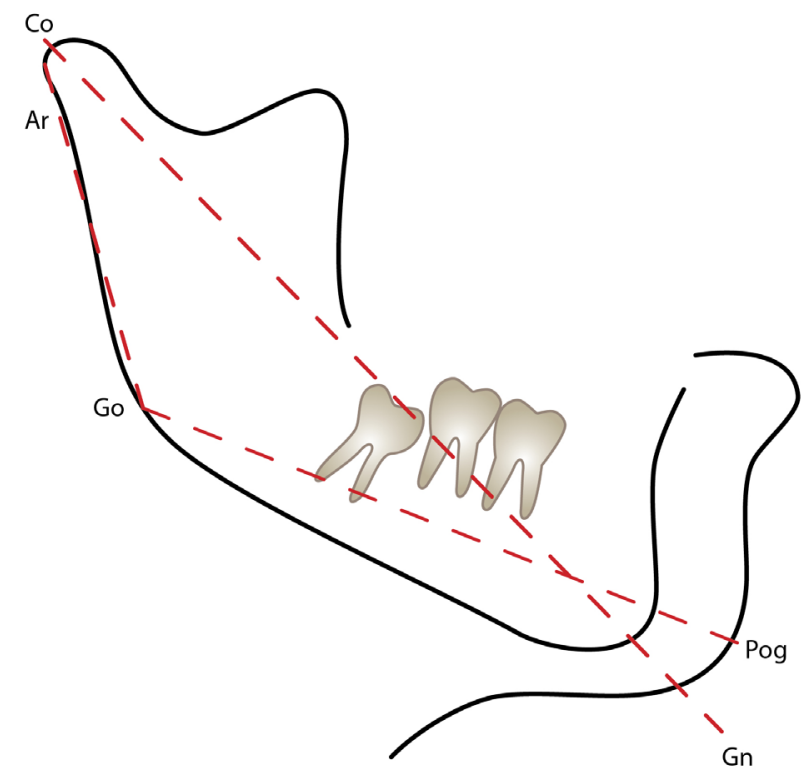

Figura 2. Análisis de la radiografía cefálica lateral según Kyrkanides S y Richter L(14). Co = Condilion, Go $=$ Gonion, $\mathrm{Pog}=$ Pogonion, $\mathrm{Gn}=$ Gnation, $\mathrm{Ar}=$ Articular. los valores de CCI para la evaluación intraexaminador fueron 0,9533 y 0,8986 .

Las medidas fueron hechas bilateralmente en ambas hemimandibulas. Para el análisis de las longitudes bilaterales mandibulares se utilizó un sistema de medición estandarizado, que registró la altura del cóndilo, rama y altura del cuerpo mandibular.

Para determinar la presencia de asimetría se utilizó el método empleado por Kjellberg y colaboradores en el que se tomaron tres distancias, derecha e izquierda, medidas y calculadas en milímetros en diferentes tiempos (13). A la medida derecha se le restó la medida izquierda, luego se dividió entre la derecha y se multiplico x 100 según la fórmula descrita por Rossi y colaboradores (15):

Tamaño de la rama derecha - tamaño de la rama izquierda x 100 Tamaño de la rama derecha

El tamaño de la rama derecha fue utilizado como referencia, el valor negativo del tamaño de la rama izquierda no se tomó en cuenta para el análisis estadístico. La presencia de asimetría se consideró a partir de un valor mínimo de $2 \mathrm{~mm}$ que equivale a un porcentaje mayor de $2,8 \%$ (15).

La base de datos se elaboró en Excel por duplicado y se exportó al paquete estadístico Epi-Info 6-04. Para comparar las mediciones radiográficas entre los dos grupos de estudio, se aplicó la prueba t de Student y el análisis de varianza (ANOVA). Se consideró un valor de $\mathrm{p} \leq 0,05$ como estadísticamente significativo. Este trabajo se acogió a la Resolución 08430 de 1993 del Ministerio de Salud de la República de Colombia en la que se estipulan las normas técnicas, académicas y administrativas para la investigación en salud al clasificarse como una "investigación sin riesgo" por ser un estudio científico en el que no se realizó modificación intencionada de las variables biológicas, fisiológicas, psicológicas o sociales de los individuos. Adicionalmente, fue aprobado por el Comité de Ética en Investigación de la Facultad de Odontología de la Universidad Santo Tomás en Bucaramanga.

\section{RESULTADOS}

La muestra estuvo constituida por radiografías panorámicas y cefálicas laterales de 130 pacientes, 65 
(50,0\%) correspondían al grupo con labio y/o paladar hendido (LPH). El promedio de edad fue de 10,8 \pm 3,2 años en los niños que presentaban hendiduras y de $10,3 \pm 2,9$ en los que no las presentaban. No se observó una diferencia estadísticamente significativa en la edad según la presencia de LPH $(\mathrm{p}=0,3982)$.

Al evaluar la presencia de asimetría se encontró una diferencia estadísticamente significativa de mayor asimetría en pacientes con labio y/o paladar hendido respecto a grupo sin labio $\mathrm{y} / \mathrm{o}$ paladar hendido $(\mathrm{p}=0,0340)$ (Tabla 1$)$.

El tamaño del cuerpo mandibular representado por Go-Pog, Co-Gn en las radiografías de los pacientes con LPH fue menor al compararlo con las radiografías de los pacientes sin hendidura. Se encontró una diferencia estadísticamente significativa en el tamaño del cuerpo mandibular, representado en las medidas Go-Pog $(\mathrm{p}=0,0047)$ y Co-Gn $(\mathrm{p}=0,0395)$ según los grupos (Tabla 2).

Se encontró una diferencia estadísticamente significativa en la simetría de las radiografías de los pacientes con labio y paladar hendido, así como una menor altura en la longitud de la rama mandibular izquierda representada por el plano cefalométrico Co-Inc (Tabla 3). También, se observó una diferencia estadísticamente significativa en el tamaño del cuerpo mandibular en todas las medidas (Tabla 4).

Tabla 1. Descripción de las variables sociodemográficas analizadas en forma global y según la presencia de LPH.

\begin{tabular}{lllll}
\hline Variables & $\begin{array}{l}\text { Global } \\
\mathbf{n}(\%)\end{array}$ & $\begin{array}{l}\text { Labio y/o paladar } \\
\mathbf{n}(\mathbf{\%})\end{array}$ & $\begin{array}{l}\text { Control } \\
\mathbf{n}(\mathbf{\%})\end{array}$ & P \\
\hline $\begin{array}{l}\text { Sexo } \\
\text { Masculino }\end{array}$ & $64(49,2)$ & $37(57,8)$ & $27(42,2)$ & 0,0790 \\
Femenino & $66(50,8)$ & $28(42,4)$ & $38(57,6)$ & \\
Asimetría & & & & 0,0340 \\
Con asimetría & $43(33,1)$ & $30(46,2)$ & $13(20,0)$ & \\
Sin asimetría & $87(66,9)$ & $35(53,8)$ & $52(80,0)$ & \\
\hline
\end{tabular}

Tabla 2. Descripción de las medidas de crecimiento y desarrollo mandibular en radiografías de pacientes con y sin LPH.

\begin{tabular}{llll}
\hline Variables & $\begin{array}{l}\text { Labio y/o paladar } \\
\mathbf{X} \pm \mathbf{D E}\end{array}$ & $\begin{array}{l}\text { Control } \\
\mathbf{X} \pm \mathbf{D E}\end{array}$ & $\mathbf{P}$ \\
\hline Ar - Go & $39,6 \pm 5,6$ & $41,2 \pm 4,9$ & 0,0888 \\
Go - Pog & $68,8 \pm 6,4$ & $72,0 \pm 6,1$ & 0,0047 \\
Co - Gn & $105,7 \pm 9,7$ & $109,0 \pm 8,3$ & 0,0395
\end{tabular}

X: promedio. DE: Desviación Estándar. Las medidas son mostradas en milímetros.

Tabla 3. Descripción de las variables relacionadas con el tamaño de las alturas derecha e izquierda en radiografías de pacientes con y sin LPH.

\begin{tabular}{llll}
\hline Variables & $\begin{array}{l}\text { Labio y/o paladar } \\
\mathbf{X} \pm \mathbf{D E}\end{array}$ & $\begin{array}{l}\text { Control } \\
\mathbf{X} \pm \mathbf{D E}\end{array}$ & $\mathbf{P}$ \\
\hline Co - Inc der. & $18,2 \pm 3,1$ & $19,1 \pm 3,0$ & 0,1503 \\
Co - Inc izq. & $18,5 \pm 3,5$ & $19,8 \pm 3,1$ & 0,0414 \\
Inc - Go der. & $39,7 \pm 5,9$ & $39,5 \pm 5,3$ & 0,8894 \\
Inc - Go izq. & $39,3 \pm 5,7$ & $39,2 \pm 5,1$ & 0,9358 \\
Co - Go der. & $58,0 \pm 6,6$ & $58,5 \pm 5,8$ & 0,6457 \\
Co - Go izq. & $57,8 \pm 6,6$ & $59,0 \pm 6,1$ & 0,2841 \\
\hline
\end{tabular}

X: promedio. DE: Desviación Estándar. Las medidas son mostradas en milímetros 
Tabla 4. Descripción de las medidas de crecimiento y desarrollo mandibular según el tipo de hendidura comparado con radiografías de pacientes sin LPH.

\begin{tabular}{lllll}
\hline Variables & $\begin{array}{l}\text { Unilateral } \\
\mathbf{X} \pm \mathbf{D E}\end{array}$ & $\begin{array}{l}\text { Bilateral } \\
\mathbf{X} \pm \mathbf{D E}\end{array}$ & $\begin{array}{l}\text { Sanos } \\
\mathbf{X} \pm \mathbf{D E}\end{array}$ & $\mathbf{P}$ \\
\hline $\mathrm{Ar}-\mathrm{Go}$ & $40,8 \pm 6,0$ & $38,0 \pm 4,8$ & $41,2 \pm 4,9$ & 0,0260 \\
$\mathrm{Go}-\mathrm{Pog}$ & $70,4 \pm 6,8$ & $66,8 \pm 5,3$ & $72,1 \pm 6,1$ & 0,0015 \\
$\mathrm{Co}-\mathrm{Gn}$ & $107,8 \pm 10,2$ & $102,7 \pm 8,3$ & $109,0 \pm 8,3$ & 0,0094 \\
\hline
\end{tabular}

$\mathrm{X}$ : promedio. DE: Desviación Estándar. Las medidas son mostradas en milímetros

\section{DISCUSIÓN}

El presente estudio, en población colombiana, demostró una diferencia estadísticamente significativa $(\mathrm{p}=0,034)$ de mayor asimetría en las radiografías de pacientes con labio y paladar hendido respecto a las radiografías de pacientes sin la hendidura. Estos hallazgos coinciden con los encontrados en otras razas $(8,12)$; lo que demostraría la relación del genotipo, sobre toda la expresión fenotípica a nivel craneofacial, y no solo a nivel maxilar, donde se hace mas evidente por la presencia de la hendidura.

La asimetría facial ha sido tema de estudio en individuos normales; varias publicaciones coinciden en encontrar asimetría facial como un hallazgo frecuente en las diferentes poblaciones, al ser la cara humana, en sus lados derecho e izquierdo de diferente forma $(16,17)$. Generalmente, estos hallazgos se evidencian con el uso de radiografías posteroanteriores y mediante análisis que evalúan cuantitativamente las dimensiones vertical, transversal y sagital del complejo craneofacial (17). Posiblemente, estas diferencias en el desarrollo son muy pequeñas para ser medidas, siendo menores de $1 \mathrm{~mm}$, lo que para muchos autores, es considerada una asimetría normal (18). No obstante, el uso rutinario de la cefalometría posteroanterior es limitado por la dificultad de identificación de los puntos, además de no ser una radiografía de rutina para la consulta odontológica. Pirttiniemi, y Lemos y colaboradores reseñaron que la radiografía panorámica también es un método útil para estudiar la simetría de la rama y el cóndilo de la mandíbula por separado en los lados derechos e izquierdos, particularmente en las medidas verticales $(19,20)$.

En varias publicaciones, la radiografía panorámica ha sido utilizada para estudiar asimetría facial. Kirkanides y Ritcher señalaron pertinente este uso dado que normalmente son obtenidas de manera rutinaria en los pacientes pediátricos; además, este tipo de examen radiográfico puede ser utilizado como un indicador fiable de la asimetría mandibular o facial, sin la exposición de los pacientes pediátricos a una radiación innecesaria (14). Rossi y colaboradores propusieron un índice de asimetría en el que el tamaño mandibular del lado derecho se le resta el lado izquierdo, se divide por el lado derecho y se multiplica por cien; no se tiene en cuenta el signo negativo. Estos autores reportaron la presencia de asimetría en toda la muestra con un valor mínimo de 2,8\% y máximo de 6,5\% (15). Un índice similar se aplicó en un estudio realizado por Sağlam en 2003 en donde se utilizó el lado izquierdo y se siguió el mismo parámetro del estudio anterior (21). En el presente estudio se utilizó la radiografía panorámica por formar parte del paquete básico utilizado por todas las facultades de odontología y odontólogos en su consulta privada; tener una baja radiación y ser una proyección útil para examinar las estructuras dentales y óseas del maxilar superior e inferior, al permitir determinar la presencia de una alteración macroscópica, además de comparar la forma de la rama ascendente y de los cóndilos mandibulares de ambos lados. Para la definición de la asimetría mandibular se aplicó el índice de asimetría propuesto por Rossi y colaboradores (15).

El estudio de la asimetría facial, en pacientes con hendiduras, también ha sido reportado por varios autores que han encontrado un incremento en asimetría de tipo vertical en pacientes con LPH comparados con sus controles (22-24); resultados similares se muestran en estudios recientes que utilizan como medio diagnóstico tomografías $(8,9)$.

Investigaciones en pacientes con hendiduras muestran resultados similares en cuanto la asimetría mandibular y la relación con el efecto de las cirugías y tratamientos. Smahel y colaboradores, y Cagáňová y colaboradores no encontraron diferencias 
estadísticamente significativas entre dos grupos de pacientes tratados con protocolos de tratamientos diferentes, en cuanto al tamaño y la forma de los maxilares, salvo la rama mandibular más corta en los pacientes con hendidura labio palatina unilateral completa $(25,26)$. La comparación con los grupos control reveló un cuerpo y rama mandibular más corto y la diagonal mandibular pequeña. Al igual que el estudio de da Silva y colaboradores, que concluyen que las cirugías no tienen efecto alguno sobre el crecimiento mandibular (27).

Al revisar los resultados del presente estudio y los reportes anteriores, se observa que hay un buen desarrollo de la morfología mandibular en los pacientes con labio y/o paladar hendido que no es afectada por la presencia de la hendidura labio palatina, el efecto de las cirugías, la edad o el sexo del paciente, pero sí las dimensiones mandibulares, son más pequeñas cuando se comparan con los controles, ya que se encontraron diferencias estadísticamente significativas de menor tamaño del cuerpo mandibular, representado en la medida Go-Pog $(\mathrm{p}=0,0047)$ y en la diagonal mandibular por la medida Co-Gn ( $\mathrm{p}=0,0395)$. Resultados similares los encontró Daskalogiannakis y colaboradores al observar un menor tamaño mandibular en los pacientes con LPH del Centro de Nijmegen (Holanda) en los que se observó un perfil convexo y no, en los pacientes del Centro de Toronto (Canadá), donde se encontró perfil cóncavo, aunque presentaban similitud en tamaño maxilar (28) Estos aspectos podrían sugerir que los resultados de tamaño mandibular son diferentes entre poblaciones y grupos de muestras, pues los hallazgos de esta investigación coinciden con el grupo de Nijmegen, pero difieren del Centro de Toronto.

Estas observaciones coinciden cuando se han realizado estudios poblacionales genéticos y se correlacionan la forma y asimetría facial con su genotipo (11); varios autores muestran y explican el fenotipo de la asimetría, con los respectivos genes $(29,30,31)$. Se requieren futuras investigaciones con tomografías que relacionen las dimensiones mandibulares con estudios genéticos.

Se podría concluir que el tamaño del cuerpo mandibular representado por las medidas tomadas en radiografías panorámicas y cefálicas laterales (Go-Pog, Co-Gn) de pacientes con labio y paladar hendido es menor que el de los pacientes sin hendida al hallarse una diferencia estadísticamente significativa de mayor asimetría en las radiografías de los pacientes con hendiduras.

\section{Declaración de financiamiento y de conflicto de intereses}

La investigación se financió con recursos propios.

Los autores manifiestan no tener conflictos de interés.

\section{Correspondencia:}

Ethman Ariel Torres Murillo

Calle 48 28-27 Bucaramanga, Colombia.

Teléfono: 0573174340168

Correo electrónico: ethman.torres@ustabuca.edu.co

\section{REFERENCIAS BIBLIOGRAFICAS}

1. Smarius B, Loozen C, Manten W, et al. Accurate diagnosis of prenatal cleft lip/palate by understanding the embryology. World J Methodol. 2017;26(7):93100. doi: 10.5662/wjm.v7.i3.93.

2. Beaty TH, Marazita ML, Leslie EJ. Genetic factors influencing risk to orofacial clefts: today's challenges and tomorrow's opportunities. F1000Res. 2016; 5(2800). doi: 10.12688/f1000research.9503.1

3. Burg ML, Chai Y, Yao CA, et al. Epidemiology, etiology, and treatment of isolated cleft palate. Front Physiol. 2016;1:7-67. doi: 10.3389/fphys.2016.00067

4. Krauss RS, Hong M. Gene-environment interactions and the etiology of birth defects. Curr Top Dev Biol. 2016;1(16):569-80. doi: 10.1016/bs.ctdb.2015.12.010.

5. Velázquez-Aragón JA, Alcántara-Ortigoza MA, Estandia-Ortega B, et al. Gene interactions provide Evidence for Signaling Pathways Involved in Cleft Lip/ Palate in Humans. J Dent Res. 2016;95(11):1257-64. doi: $10.177 / 0022034516647034$.

6. Tam PP, Fossat N, Wilkie E, et al. Formation of the embryonic head in the mouse: attributes of a gene regulatory network. Curr Top Dev Biol. 2016;1(17):497521. doi: 10.1016/bs.ctdb.2015.11.019.

7. Meazzini MC, Corno M, Novelli G, et al. Longterm computed tomographic evaluation of alveolar bone rormation in patients with unilateral cleft lip and palate after early secondary gingivoalveoloplasty. Plast Reconstr Surg. 2016;137(2):365e-74e. doi:10.1097/01.

8. Yang L, Chen Z, Zhang X. A cone-beam computed tomography evaluation of facial asymmetry in unilateral cleft lip and palate individuals. J Oral Sci. 2016; 58(1):109-15. doi: 10.2334/josnusd.58.109.

9. Lin $\mathrm{Y}$, Chen G, Fu Z, et al. Cone-beam computed 
tomography assessment of lower facial asymmetry in unilateral cleft lip and palate and non-cleft patients with class III skeletal relationship. PLoS One. 2015;10(8):e0130235. doi: 10.1371/journal.pone. 0130235

10. Laspos CP, Kyrkanides S, Tallents RH, et al. Mandibular asymmetry in noncleft and unilateral cleft lip and palate individuals. Cleft Palate Craniofac J. 1997;34(5):4106. doi: 10.1597/1545-1569_1997_034_0410_mainau 2.3.co_2

11. Miller SF, Weinberg SM, Nidey NL, et al. Exploratory genotype-phenotype correlations of facial form and asymmetry in unaffected relatives of children with nonsyndromic cleft lip and/or palate. J Anat. 2014;224(6):688-709. doi: 10.1111/joa.12182.

12. Manyama M, Larson JR, Liberton DK, et al. Facial morphometrics of children with non-syndromic orofacial clefts in Tanzania. BMC Oral Health. 2014;29:14:93. doi: 10.1186/472-6831-14-93.

13. Kjellberg H, Ekestubbe A, Kiliaridis S, Thilander B. Condylar height on panoramic radiographs. A methodologic study with a clinical application. Acta Odontol Scand. 1994;52(1):43-50.

14. Kyrkanides S, Richter L. Mandibular asymmetry and antigonial notching in individuals with unilateral cleft lip and palate. Cleft Palate Craniofac J. 2002;39(1):305. doi: 10.1597/1545-1569_2002_039_0030_maaani 2.0.co_2

15. Rossi M, Ribeiro E, Smith R. Craniofacial asymmetry in development: an anatomical study. Angle Orthod. 2003;73(4):381-5. doi: 043/ 0003-3219(2003)073<0381:CAIDAA $>2.0 . \mathrm{CO} ; 2$

16. Srivastava D, Singh H, Mishra S, et al. Facial asymmetry revisited: Part I- diagnosis and treatment planning. J Oral Biol Craniofac Res. 2018;8(1):7-14. doi: 0.1016/j.jobcr.2017.04.010

17. Srivastava D, Singh H, Mishra S, et al. Facial asymmetry revisited: Part II-Conceptualizing the management. J Oral Biol Craniofac Res. 2018;8(1):15-9. doi: 0.1016/j. jobcr.2017.09.008

18. Farkas LG, Cheung G. Facial asymmetry in healthy North American Caucasians. An anthropometrical study. Angle Orthod. 1981;51(1):70-7. doi: 10.1043/0003-3219(1981)051<0070:FAIHNA $>2.0$. $\mathrm{CO} ; 2$

19. Pirttiniemi PM. Associations of mandibular and facial asymmetries-a review. Am J Orthod Dentofacial Orthop. 1994;106(2):191-200. doi: 10.1016/S08895406(94)70038-9

20. Lemos AD, Katz CR, Heimer MV, Rosentblatt A. Mandibular asymmetry: a proposal of radiographic analysis with public domain software. Dental Press J Orthod. 2014;19(3):52-8.

21. Sağlam AM. The condylar asymmetry measurements in different skeletal patterns. J Oral Rehabil.
2003;30(7):738-42.

22. Ras F, Habets LL, van-Ginkel FC, Prahl-Anderesen B. Longitudinal study on three-dimensional changes of facial asymmetry in children between 4 to 12 years of age with unilateral cleft lip and palate. Cleft Palate Craniofac J. 1995; 32(6): 463-468. doi: 10.1597/1545156919950320463 lsotdc 2.3.co 2

23. Ras F, Habets LL, van Ginkel FC, Prahl-Andersen B. Three-dimensional evaluation of facial asymmetry in cleft lip and palate. Cleft Palate Craniofac J. 1994;31(2):116-121 doi: 10.1597/1545-1569 1994_031_0116_tdeofa_2.3.co_2

24. Al-Rudainy D, Ju X, Mehendale FV, Ayoub A. Longitudinal 3D Assessment of facial asymmetry in unilateral cleft lip and palate. Cleft Palate Craniofac J. 2019;56(4):495-501. doi: 10.1177/10556656187 80108.

25. Smahel Z, Müllerova Z, Nejedly A, Horak I. Changes in craniofacial development due to modifications of the treatment of unilateral cleft lip and palate. Cleft Palate Craniofac J. 1998;35(3):240-7. doi: 10. 1597/1545-1569_1998_035_0240_cicddt_2.3.co_2

26. Cagáňová V, Borský J, Smahel Z, Veleminská J. Facial growth and development in unilateral cleft lip and palate: comparison between secondary alveolar bone grafting and primary periosteoplasty. Cleft Palate Craniofac J. 2014;51(1):15-22. doi: 10.1597/11-174

27. da Silva Júnior OG, Normando AD, Capelozza Júnior L. Mandibular morphology and spatial position in patients with clefts: intrinsic or iatrogenic? Cleft Palate Craniofac J. 1992;29(4):369-75. doi: 10.1597/1545-1569_1992_029_0369_mmaspi 2.3.co 2

28. Daskalogiannakis J, Dijkman GE, Kuijpers-Jagtman AM, Ross RB. Comparison of facial morphology in two populations with complete unilateral cleft lip and palate from two different centers. Cleft Palate Craniofac J. 2006;43(4):471-6. doi: 10.1597/05-082.1

29. Weinberg SM, Cornell R, Leslie EJ. Craniofacial genetics: Where have we been and where are we going? PLoS Genet. 2018;21(14):e1007438. doi:10.1371/ journal.pgen.1007438.

30. Sun Z, da Fontoura CSG, Moreno M, et al. FoxO6 regulates Hippo signaling and growth of the craniofacial complex. PLoS Genet. 2018;14 (10):e1007675. doi:10.1371/journal.pgen.1007675.

31. Claes P, Roosenboom J, White JD, et al. Genomewide mapping of global-to-local genetic effects on human facial shape. Nat Genet. 2018;50(3):414-23. doi: 10.1038/s41588-018-0057-4.

Recibido : 29-01-2021

Aceptado : 22-04-2021 